\section{Genes, pathways, and animal models in primary open-angle glaucoma}

\author{
Abstract \\ Glaucoma is an optic neuropathy characterized \\ by loss of retinal ganglion cells (RGCs) and \\ consequently visual field loss. It is a complex \\ and heterogeneous disease in which both \\ environmental and genetic factors play a role. \\ With the advent of genome-wide association \\ studies (GWASs), the number of loci asso- \\ ciated with primary open-angle glaucoma \\ (POAG) have increased greatly. There has also \\ been major progress in understanding the \\ genes determining the vertical cup-disc ratio \\ (VCDR), disc area (DA), cup area (CA), \\ intraocular pressure (IOP), and central corneal \\ thickness (CCT). In this review, we will update \\ and summarize the genetic loci associated so \\ far with POAG, VCDR, DA, CA, IOP, and \\ $\mathrm{CCT}$. We will describe the pathways revealed \\ and supported by genetic association studies, \\ integrating current knowledge from human \\ and experimental data. Finally, we will discuss \\ approaches for functional genomics and \\ clinical translation. \\ Eye (2015) 29, 1285-1298; doi:10.1038/eye.2015.160; \\ published online 28 August 2015

\section{Introduction} \\ Glaucoma is a complex, heterogeneous disease \\ and the leading cause of irreversible blindness \\ worldwide. In 2013, 64.3 million people were \\ affected by glaucoma, and it is expected that \\ 76 million will be affected in 2020. ${ }^{1}$ Primary \\ open-angle glaucoma (POAG), the most \\ common form of glaucoma in all populations, \\ is characterized by visual field loss because of \\ progressive death of retinal ganglion cells \\ (RGCs). The known risk factors for POAG \\ include a higher age, African ancestry, high \\ intraocular pressure (IOP), decreased central \\ corneal thickness (CCT), high myopia, and a \\ positive family history for glaucoma. ${ }^{2-4}$ There is \\ growing evidence that diabetes mellitus and \\ hypertension, both components of the metabolic \\ syndrome, may be potential risk factors. ${ }^{2}$
}

Al Iglesias 1,2, H Springelkamp ${ }^{1,3}$, WD Ramdas ${ }^{3}$, CCW Klaver ${ }^{1,3}$, R Willemsen ${ }^{2}$ and CM van Duijn ${ }^{1}$

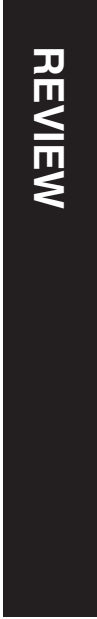

The genetic contribution to POAG has long been recognized. First-degree relatives of glaucoma patients are estimated to have a 10-fold increased risk of POAG compared with the general population. ${ }^{5}$ Various disease-causing mutations in optineurin (OPTN), myocilin (MYOC), and WD-repeat domain 36 (WDR36) genes have been long identified as the cause of familial forms of POAG. ${ }^{6}$ In the past decade, genome-wide association studies (GWASs, see Figure 1 for details) have successfully identified over 70 single-nucleotide polymorphism (SNPs) associated with POAG and related quantitative traits or endophenotypes. The description of the candidate genes tagged by these SNPs is shown in Supplementary Table 1.

Endophenotypes are measurable traits with a strong genetic component that are related to a disease, POAG in this case (see Figure 2). Complex diseases, such as POAG, are generally linked to various (in)dependent endophenotypes and vice versa; one endophenotype can be related to multiple other diseases apart from POAG. Endophenotypes for POAG include IOP, CCT, cup area (CA), vertical cup-disc ratio (VCDR), and disc area (DA). The genetic component of endophenotypes is usually quantified by the heritability, a proportion that ranges from 0 (no genetic effect) to 1 (a phenotype that is completely determined by genes). The heritability of POAG endophenotypes is very high: 0.55 for IOP, 0.85 for CCT, 0.72 for DA, 0.75 for CA, and between 0.48 and 0.66 for VCDR. ${ }^{7}$

Here we review the genes that GWASs have brought to surface in the context of the question to what extent GWASs have improved the understanding of the specific pathways and biological processes implicated in the pathophysiology of POAG. This review aims to integrate current knowledge from human and experimental data in POAG. First, we will provide an update of the loci associated with POAG and its endophenotypes and then we will
${ }^{1}$ Department of Epidemiology, Erasmus University Medical Center, Rotterdam, The Netherlands

${ }^{2}$ Department of Clinical Genetics, Erasmus University Medical Center, Rotterdam, The Netherlands

${ }^{3}$ Department of Ophthalmology, Erasmus University Medical Center, Rotterdam, The Netherlands

Correspondence: CM van Duijn, Department of Epidemiology, Erasmus Medical Center, PO Box 2040, 3000 CA Rotterdam, The Netherlands Tel: +31 107043 394; Fax: +31 107044657 . E-mail: c.vanduijn@ erasmusmc.nl

Received: 5 May 2015 Accepted in revised form: 27 July 2015 Published online: 28 August 2015 


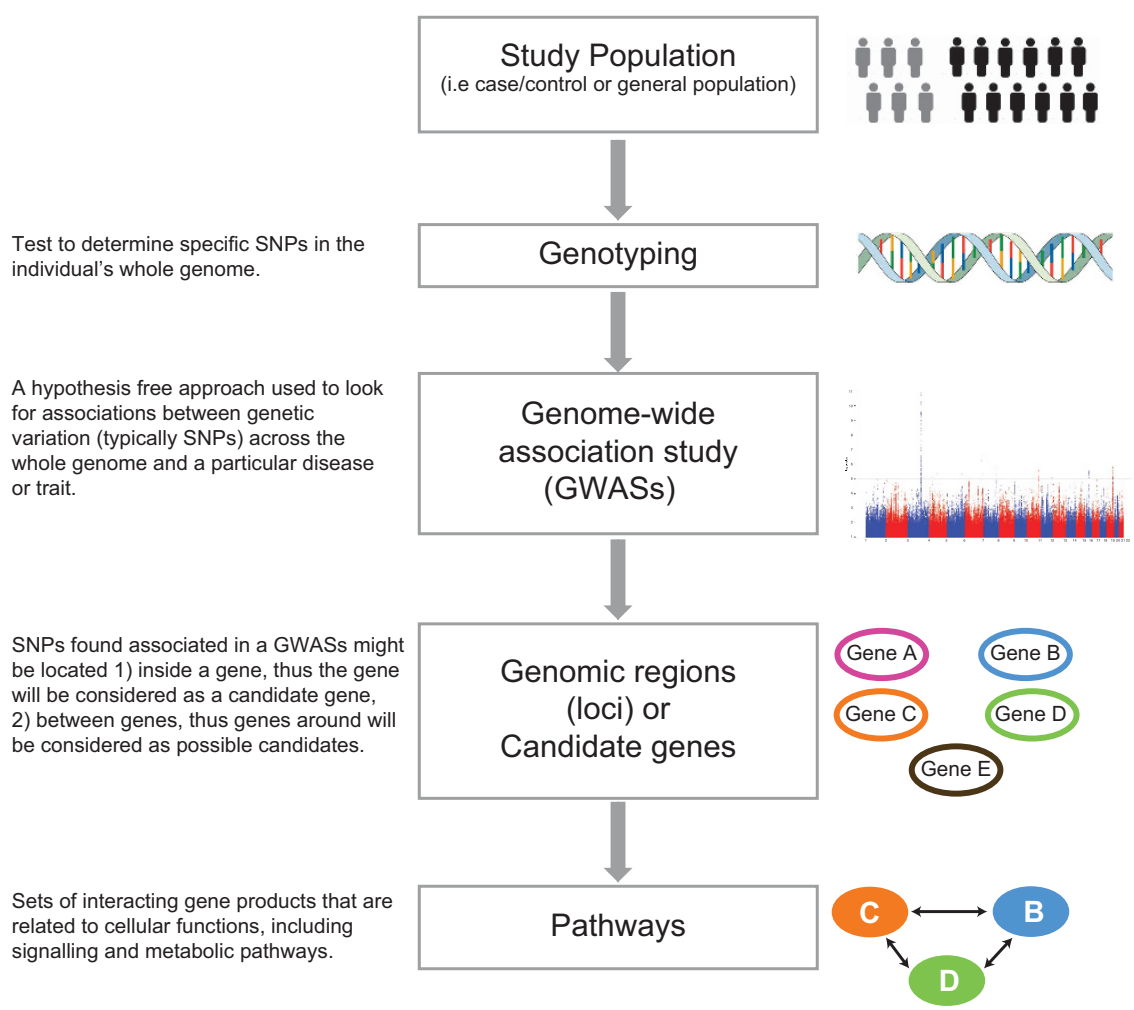

Figure 1 Schematic representation of the stages involved in genome-wide association studies (GWASs). GWASs are based on either a case/control or a population-based design in which single-nucleotide polymorphisms (SNPs) across the whole genome are genotyped. Outcome of GWASs are associated SNPs that are tagging candidate genes or genomic regions (loci). Identified genes may lead to identification of pathways involved in the disease.

describe the molecular pathways and biological functions pointed by genetic association studies. Furthermore, we will evaluate the role of animal models for this disease in the era of functional genomics. Finally, we will discuss the potential uses of GWASs findings in the clinical practice.

\section{Search strategy}

We searched the MEDLINE/PubMed database for articles from December 2009 to March 2015. Search terms covered the keywords: 'glaucoma', 'genetic association studies', 'single nucleotide polymorphism', 'animal models', and 'pathway'. A manual search was also based on references from retrieved articles.

\section{Rare and common variants associated with POAG}

Linkage studies have identified at least 20 genomic regions linked to POAG. In three of these genomic regions, the causal genes have been identified: $M Y O C_{,}^{8} O P T N,{ }^{9}$ and WDR36. ${ }^{10}$ In addition, copy number variations in TBK1 gene were linked to normal-tension glaucoma (NTG) in an African-American pedigree ${ }^{11}$ that was confirmed in Caucasians and Asians populations. ${ }^{12-14}$ Mutations in MYOC, OPTN, and WDR36 account for $<10 \%$ of POAG cases, ${ }^{15}$ and copy number variations in TBK1 explained 0.4 to $1.3 \%$ of NTG cases. $^{12}$ POAG is a complex disease in which multiple genetic variants with small effects and environmental factors may play a role. GWASs is a powerful approach to identify common SNPs or genetic variants with modest effects, and have consistently identified 13 genetic regions as glaucoma susceptibility loci: ATOH7, CAV1/CAV2, CDKN2B/CDKN2B-AS1, GAS7, SIX6, $T M C O 1$, a regulatory region at $8 \mathrm{q} 22$, and recently $A B C A 1$, AFAP1, ARHGEF12, CDC7/TGF $\beta R 3$, GMDS, and PMM2 were added to the list. ${ }^{16-25}$ In addition to SNPs, copy number variants (ie, submicroscopic chromosomal deletions and duplications) have been shown to play a role in POAG. Besides copy number variations in TBK1, genomic duplications (in CDKN2B-AS1, near GAS7 and TMCO1) and deletions (in SIX6 and $A T O H 7$ ) have been reported in POAG cases. In Figure 3 the genes associated with POAG in general, NTG, and high-tension glaucoma (HTG) are shown.

\section{Common variants associated with POAG endophenotypes}

Research of endophenotypes has been a successful strategy to elucidate the genetics of POAG. 
a

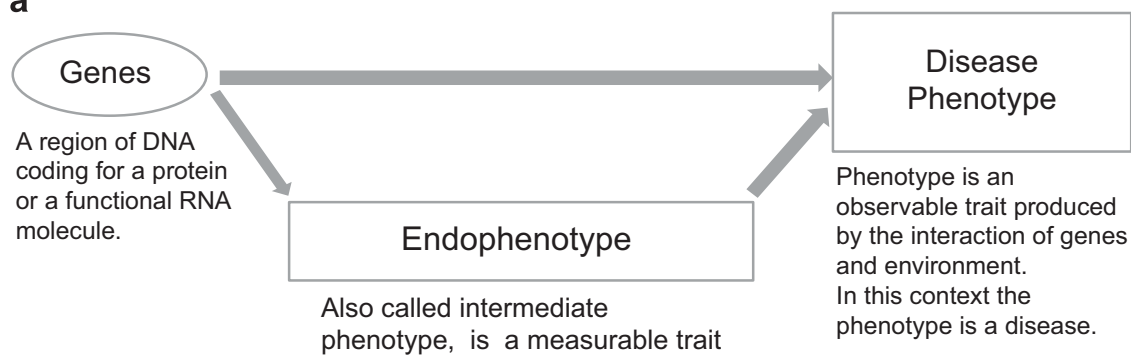

with a strong genetic component,

which associates with a disease

but is not itself a symptom of the

disease.

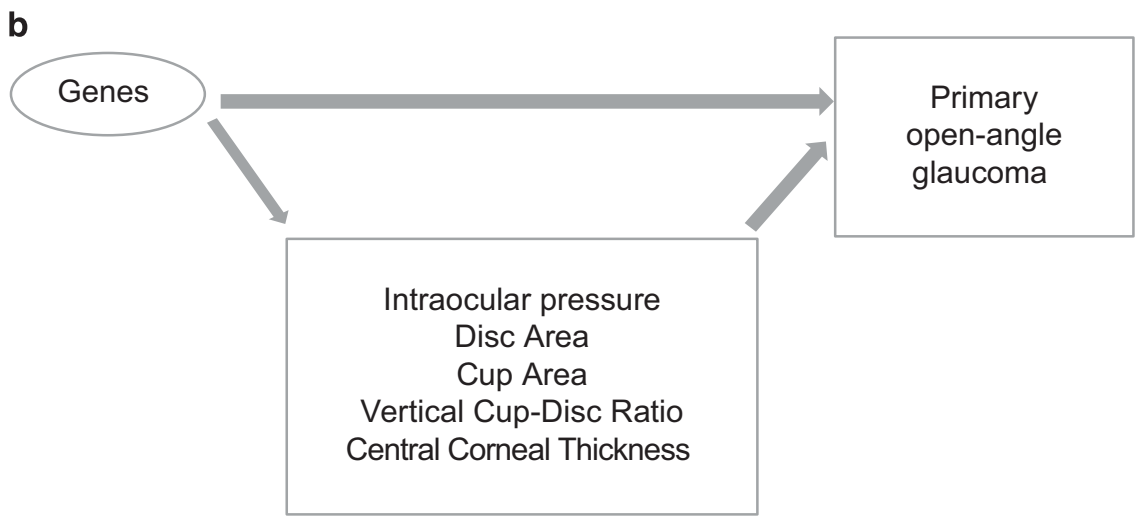

Figure 2 Endophenotype. (a) Definition and relation of endophenotype, phenotype, and gene. (b) Use of endophenotype in the context of primary open-angle glaucoma.

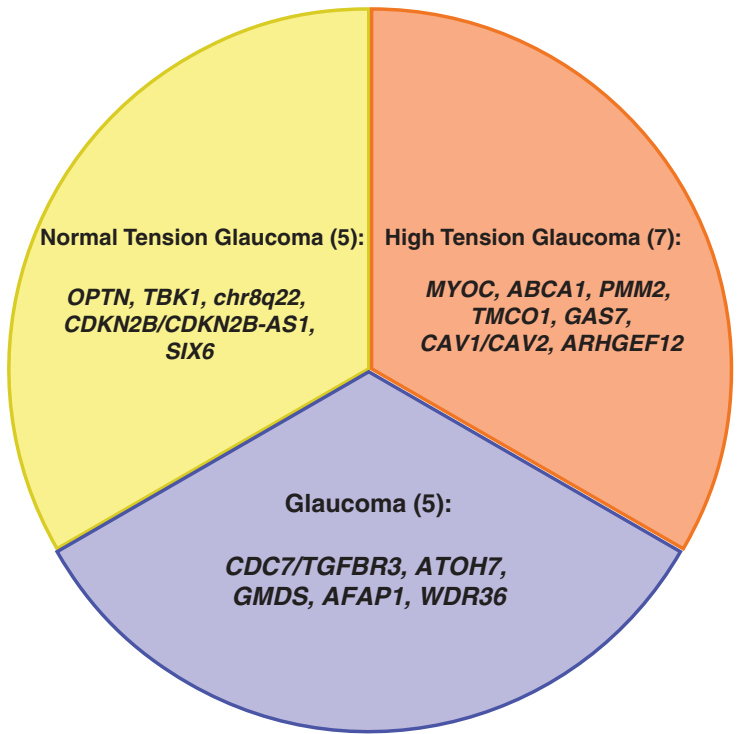

Figure 3 Genes implicated in POAG. The pie diagram illustrates the number of loci that show association with POAG including the familial forms. Three big groups are defined based on whether loci show association with normal-tension glaucoma, high-tension glaucoma, or POAG in general.
The endophenotype strategy allows individuals to be ranked along the continuum of risk as opposed to ranking individuals as patients and controls. Selection of controls in a late-onset disease like POAG can be difficult because of the fact that any control may later become a case. Figure 4 shows the variants identified so far for optic disc parameters (VCDR, CA, and DA), IOP, and CCT in GWASs.

\section{Optic nerve parameters}

A total of 38 loci have been found associated with optic disc parameters. Of these, 18 loci have been found for VCDR: ADAMTS8, ATOH7, BMP2, CDC7/TGF $\beta$ R3, CARD10, CDKN2B/CDKN2B-AS1, CHEK2, COL8A1, DUSP1, EXOC2, HSF2, PLCE1, RPAP3, SSSCA1, SIX1/6, SALL1, TMTC2, and RERE. ${ }^{26-28}$ For DA, 14 loci have been identified, including 5 loci also associated with VCDR (ATOH7, CARD10, CDC7/TGFBR3, SALL1, and TMTC2) and 9 exclusively DA loci: $A B I 3 B P, C D C 42 B P A$, DCAF4L2, F5, DIRC3, ELP4, HORMAD2, NR2F2, and $R A R B .{ }^{26,29,30}$ Finally, for CA, 22 loci have been found, including 11 loci overlapping with VCDR. VCDR and CA are two highly correlated endophenotypes $(r=0.78),{ }^{30}$ and thus the large overlap between genetic variants is 
a

Genes implicated in POAG and

Optic Disc Parameters

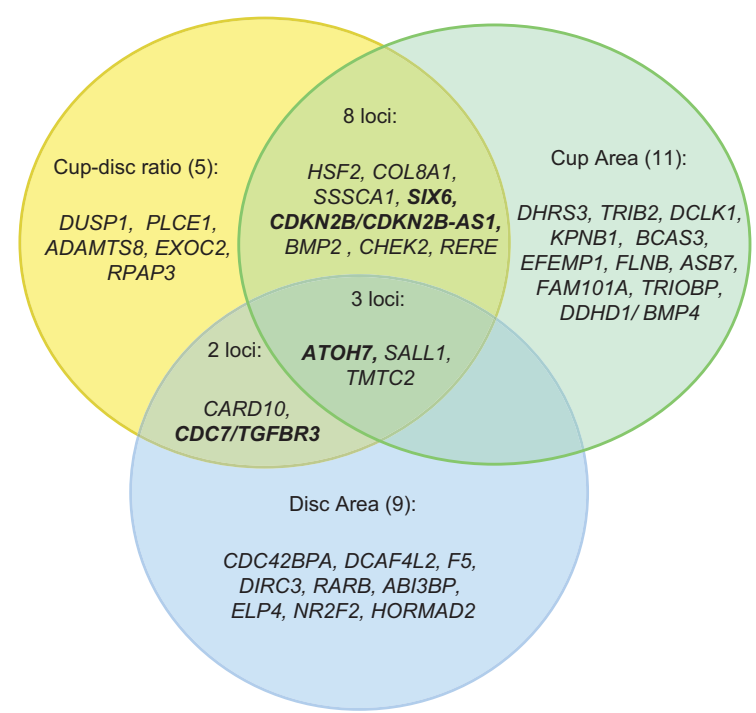

b
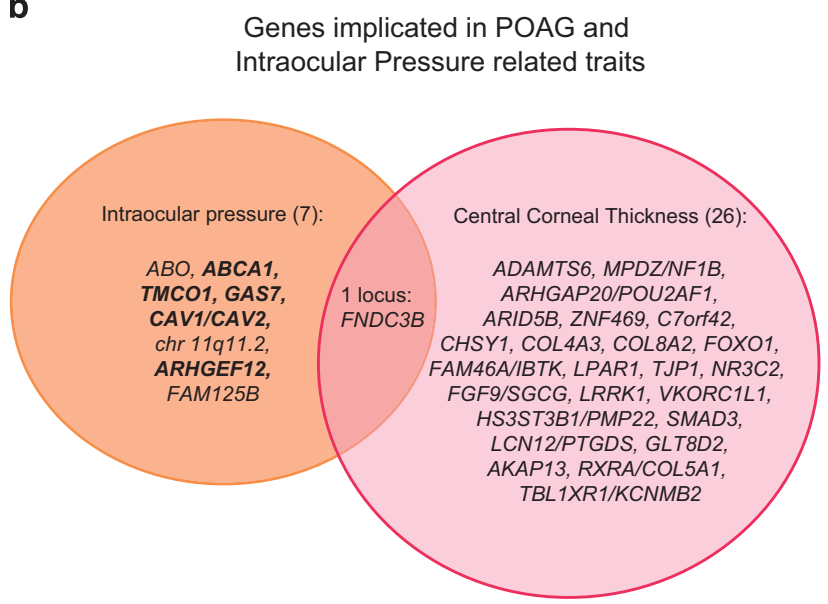

Figure 4 Genes implicated in glaucoma and endophenotypes. The Venn diagram illustrates the number of loci that show association with (a) optic disc parameters and (b) intraocular pressure and central corneal thickness. The number of loci associated with only one trait is mentioned in parentheses after the trait name, and genes names are listed below. Loci that show association with two or more traits are shown in the appropriate segment. Genes in bold have also been associated with POAG.

expected (see Figure 4a). In the case of VCDR, there are 5 loci associated with CA that have not reached yet genome-wide significance. For CA there are 11 loci not shared with VCDR: ASB7, BCAS3, DCLK1, DDHD1/BMP4, DHRS3, EFEMP1, FAM101, FLNB, KPNB1, TRIB2, and TRIOBP ${ }^{30}$ (see Figure 4a). These $\mathrm{CA}$-associated loci are not genome-wide significant yet with VCDR that may be attributable to a lack of power. Only larger study sizes can overcome this.

Among the optic nerve parameters (VCDR, CA, and DA) there are three overlapping loci, shown in Figure 4a. These loci include ATOH7, SALL1, and TMTC2. The first one is a key player in the differentiation of RGCs, and long known for its role in the determination of the disc size rather than the cup size or VCDR. ${ }^{29,31}$ SALL1 is involved in development ${ }^{32}$ and TMTC2 is implicated in calcium homeostasis in the endoplasmic reticulum. ${ }^{33}$

\section{IOP and CCT}

So far, nine loci have been found associated with IOP: TMCO1, FNDC3B, CAV1/CAV2, ABCA1, ABO, ARHGEF12, FAM125B, GAS7, and a region of $\sim 3 \mathrm{Mb}$ at 11 p11.2 containing multiple genes ${ }^{21,34-36}$ (Figure $4 b$ ). Variants in TMCO1, CAV1/CAV2, and ABCA1 were found first in POAG studies and subsequently associated with IOP. ${ }^{16,17,19,20}$ CCT is a risk factor for POAG in individuals with high IOP, and over 26 loci have been identified. ${ }^{37}$ So far, the genetic overlap in loci involved in
IOP and CCT is limited. Only one gene is overlapping with IOP (ie, $F N D C 3 B$ ) that regulates cell motility and appears to activate the TGF- $\beta$ pathway in cancer. ${ }^{38}$

\section{From endophenotypes to glaucoma}

Analyses of quantitative traits in glaucoma have proven to be fruitful: seven loci found in association studies of endophenotypes showed a significant genome-wide association with POAG. Of these, four were found in association studies of optic disc parameters $A T O H 7$, CDC7/TGF $\beta R 3, C D K N 2 B / C D K N 2 B-A S 1$, and SIX6, ${ }^{26,28-30}$ representing $10.5 \%$ of the loci identified for optic disc parameters by GWASs. Although the overall number of genetic variants involved in IOP is much lower $(n=9)$, 3 out of the $9(33.3 \%)$ are also involved in POAG: $A B C A 1$, ARHGEF12, and GAS7. ${ }^{21,34,35}$ There is no evidence to date that GWASs has reached its limits. Expanding the sample size virtually always leads to identification of new variants. Furthermore, improvements in sequencing technologies and the emerging of expanded reference panels as 1000 genomes is expected to increase the number of variants in the coming years.

\section{Pathways and biological mechanisms revealed by GWASs}

Genetic variants identified through GWASs opened new perspectives in the understanding of known potential 
pathways involved in the pathogenesis of POAG. In a recent review, four pathways were described on the basis of genes discovered up to $2013:^{39}$ (1) extracellular matrix metabolism (ECM), (2) transforming growth factor- $\beta$ (TGF- $\beta$ ) signalling, (3) tumour necrosis factor $\alpha$ (TNF- $\alpha$ ) signalling, and (4) oestrogen metabolism (here integrated with 'vascular tone pathway'). In the next section, we will discuss how the new discovered genes contribute to these and other pathways.

\section{Extracellular matrix}

The ECM, a key component of multicellular organisms, is mainly composed of proteoglycans and fibrous proteins such as collagens, elastins, and fibronectins. It provides structural support for cells and participates in the regulation of many cellular functions. ${ }^{40}$ The ECM is a highly dynamic structure that undergoes controlled remodelling in response to environmental stimuli or physiological challenges, such as elevated IOP. ${ }^{40}$ Changes in the ECM composition and turnover are speculated to play an important role in the outflow resistance in the trabecular meshwork (TM) that leads to high IOP and to the fibrotic phenotype observed at the optic nerve head, particularly in the lamina cribrosa region, of glaucomatous eyes. ${ }^{40,41}$

There are no genetic variants genome-wide significantly associated with POAG linked directly to the ECM. However, various genetic variants in genes encoding collagens, key players in the ECM metabolism, are genome-wide significantly associated with the endophenotypes; COL4A3, COL5A1, and COL8A2 have been found associated with $\mathrm{CCT}^{37}$ and variants in COL8A1 with CA and VCDR. ${ }^{28,30}$ Mutations in different members of the ADAMTS proteins, involved in ECM remodelling, have been found in human and animal studies. Mutations in ADAMTS10 were found in a dog model of POAG, whereas mutations in ADAMTS10 and ADAMTS17 cause glaucoma, myopia, and ectopic lentis in humans. ${ }^{42}$ Genetic variants in ADAMTS8 have been associated with VCDR, whereas variants in ADAMTS6 have been associated with $\mathrm{CCT},{ }^{37}$ supporting a role of ECM in both IOP regulation and optic nerve degeneration.

\section{Transforming growth factor- $\beta$}

The TGF- $\beta$ and its signalling effectors have been previously implicated in glaucoma. ${ }^{43,44}$ Of the three TGF- $\beta$ isoforms (TGF- $\beta 1$, TGF- $\beta 2$, and TGF- $\beta 3$ ), TGF- $\beta 2$ is the predominant form in the eye. ${ }^{45,46}$ Analyses of the aqueous humour $(\mathrm{AH})$ of glaucoma patients have consistently found increased levels of TGF- $\beta 2$ in both $\mathrm{AH}$ and $\mathrm{TM}^{47}$ leading to an increase in ECM protein deposition in the TM and a rise in IOP ${ }^{48}$ Furthermore, elevation of TGF- $\beta 2$ has also been found in the optic nerve head of glaucoma patients ${ }^{49}$ that may induce ECM changes in the lamina cribrosa that cause deprivation of the neurotrophic factors required for the maintenance of ganglion cells. ${ }^{50}$ Bone morphogenetic proteins (BMPs) represent a subfamily of the TGF- $\beta$ superfamily. It has been shown that increased levels of TGF- $\beta 2$ inhibits BMP4 and/or BMP7, counterbalancing the effect of TGF- $\beta 2 .^{51}$

Genetic variants in TGF- $\beta$ have not been associated with POAG yet. Instead, variants near to TGF- $\beta R 3$ have been found associated with VCDR, DA, and POAG in GWASs. TGF- $\beta R 3$ is a coreceptor for the TGF- $\beta$ superfamily; it binds to all three TGF- $\beta$ isoforms and is considered necessary for TGF- $\beta 2$ signal transduction. ${ }^{43,52}$ Furthermore, two members of the BMP subfamily have been implicated into POAG endophenotypes: BMP2 (VCDR and CA) and BMP4 (CA). The BMP4 gene plays a role in optic cup morphogenesis, and is essential for lens formation. In humans, deletions of chromosome $14 \mathrm{q}$ including $B M P 4$ have been identified in patients with anophthalmia. Furthermore, $C D K N 2 B$, one of the first genes implicated in POAG and VCDR/CA by GWASs, is induced by TGF- $\beta .^{53}$

\section{TNF- $\alpha$ and ubiquitination}

The TNF- $\alpha$ signalling pathway has been implicated as mediator of neurodegeneration in POAG. Increased levels of TNF- $\alpha$ have been found in the retina and optic nerve head of glaucomatous eyes. ${ }^{54}$ Two genes involved in POAG, OPTN and TBK1, are activated in response to TNF- $\alpha$. Up until now, no other genetic variants implicated in TNF- $\alpha$ signalling have been associated with POAG. Of interest, these two genes play a key role in amyotrophic lateral sclerosis (ALS), in which the role of OPTN was long recognized but for which rare mutations in TBK1 were recently identified. ${ }^{55}$ TBK1 is involved in neuroinflammation and interacts with OPTN that is involved in autophagy. OPTN and TBK1 seem to be critical components of the pathway required for removal of pathological ribonucleoprotein inclusions. ${ }^{55}$ Dysfunction of these genes may lead to protein aggregates that is one of the main characteristic of neurodegenerative disorders including ALS, Alzheimer's, Parkinson's, and Huntington's diseases. In addition LRRK1, a gene implicated in CCT, is involved in Parkinson's disease.

Degradation of misfolded and aggregated proteins is important in cellular homeostasis, not only as protein quality control but also for the regulation of protein levels. This links POAG to the main pathway involved in protein degradation: the ubiquitin-proteasome system (UPS). The UPS is responsible for the elimination of 
misfolded or damaged proteins, and is implicated in important cellular processes including cell cycle, differentiation, proliferation, development, and synaptic plasticity. ${ }^{56}$ In the eye, ubiquitin is present in RGCs and the retinal pigmented epithelium. ${ }^{56}$ Post-translational processing of two known glaucoma genes, OPTN and MYOC, involves UPS. ${ }^{57-59}$

The most common mutation in OPTN that causes glaucoma (E50K) leads to malfunction of the UPS, ${ }^{57}$ and subsequently to apoptosis in cultured cells. ${ }^{58}$ In a study using induced pluripotent stem cells (iPSCs) from NTG patients with the E50K mutation, it was demonstrated that the E50K mutant OPTN is insoluble, generating abnormal protein deposits in the retina. Insolubility of the E50K mutant protein was attributed to a strong interaction between TBK1 and mutant OPTN that in wildtype conditions only occurs under infection-response processes. ${ }^{60}$ Interestingly, copy number variants in TBK1 have also been associated with NTG. ${ }^{11-14}$ The mechanism of the OPTN-TBK1 cross-talk and its role in neurodegeneration as observed in POAG and ALS needs to be elucidated. Alterations of the UPS have also been observed when MYOC is overexpressed or mutated. ${ }^{59}$ Of note is that mutations in OPTN are associated with NTG whereas mutations in MYOC are suspected to alter $\mathrm{AH}$ outflow, ${ }^{59}$ suggesting that the UPS system is involved in both NTG and HTG.

To date, 101 disease-causing mutations have been documented in the MYOC gene (see www.myocilin. com). ${ }^{61}$ The protein encoded by MYOC is a secreted glycoprotein highly express in the TM. The proposed pathogenic mechanism involves aggregation of misfolded mutant MYOC in the TM, resulting in decreased $\mathrm{AH}$ outflow and early-onset high IOP. ${ }^{61}$ In cell culture studies it has been observed that cells with mutations in MYOC showed protein aggregation in the endoplasmic reticulum, leading to stress and death of the TM cells. It has been proposed that the reduced ability of the UPS to eliminate misfolded mutant MYOC may result in endoplasmic reticulum stress with pathological consequences. ${ }^{62}$

Six of the genetic variants associated with optic nerve degeneration (ASB7, GMDS, HSF2, OPTN, RPAP3, and TBK1), three to IOP (MYOC, PMM2, TMCO1) and one to CCT (TBL1XR1) are involved in UPS, raising the question of whether UPS failure is a key step in the (early) pathogenesis of POAG, either as an independent pathway or interacting with neuroinflammation. This implies that abnormalities of the UPS may be related not only with familial but also with general POAG. UBC seems to be a hub in pathway analysis including candidate genes identified in association studies, ${ }^{63}$ although no genetic variants in UBC have been associated yet with POAG and its phenotypes.

\section{Vascular tone}

Oestrogen metabolism has been proposed as possible mechanism involved in POAG, mainly because of the effect of the $17 \beta$-estradiol hormone in the regulation of CAV1 and NOS3. ${ }^{39}$ However, the consistent association of $C A V 1 / C A V 2$ region also points to vascular tone. $C A V 1$ and $C A V 2$ encode caveolin-1 and caveolin-2, respectively. This region has been genome-wide significantly associated with POAG and IOP, and has been implicated in dysregulation of the vascular tone particularly through their interaction with endothelial nitric oxide synthase $(\mathrm{eNOS})^{35}$ and production of nitric oxide (NO) in the vascular endothelia. NO regulates the vascular tone in ocular arteries ${ }^{64}$ and has also been implicated in $\mathrm{AH}$ outflow regulation; ${ }^{65}$ that is, high levels of NO in a mouse model lead to increased AH outflow and thus decreased IOP. ${ }^{66}$ This finding is consistent with data showing that patients with POAG have less NO production in the TM and Schlemm's canal compared with age-matched controls. ${ }^{65}$ Variants in the NOS3 gene that codes for eNOS have been found associated with HTG in women, ${ }^{67}$ POAG patients with migraines, ${ }^{68}$ and familial POAG. ${ }^{69}$ This has led to the hypothesis that it is an oestrogenrelated pathway. However, these associations have not been found yet in the large meta-analyses. Cav-1 knockout mice show several abnormalities, including reduced retinal function due to changes in retinal microenvironment ${ }^{70}$ and possible impairment in sensing mechanical forces in the retinal veins. ${ }^{71}$ Furthermore, mice show insulin resistance, alteration in lipid metabolism, and pulmonary hypertension, ${ }^{72}$ also supporting the role of caveolins in atherosclerosis and metabolic syndrome. Up until now, there are no reports about the IOP levels in these mice.

Recent studies have shown that CAV1 binds directly to ABCA1, possibly regulating the ABCA1-mediated cholesterol efflux. ${ }^{73}$ Genetic variants in $A B C A 1$ have been found associated with POAG and IOP. However, positive regulation by CAV1 on ABCA1 seems to be cell type specific. Further in-depth examination of the interaction of ABCA1 with CAV1 in eye is needed to elucidate its possible role in IOP and POAG. In particular, as the ABCA1 protein may interact with ARHGEF12 that is involved in the RhoA/ROCK pathway that is a current target for drug development.

\section{RhoA/Rho-associated kinase pathway}

The Rho family includes three members of small G-proteins (RhoA, RhoB, and RhoC). G-proteins are inactive when bound to guanosine diphosphate (GDP) and active when bound to guanosine triphosphate (GTP). Rho-associated kinases or ROCKs are serine/threonine 
kinases activated by Rho GTPases. The RhoA/ROCK pathway is known to regulate cell contractility via actinmyosin interactions, ${ }^{74}$ and its role in IOP regulation was first demonstrated in rabbit eyes exposed to a specific ROCK inhibitor. ${ }^{75}$ Inhibition of ROCK caused a significant reduction in IOP and an increase in $\mathrm{AH}$ outflow. ${ }^{75}$ In the past years, selective ROCK inhibitors have been proposed as a new category of IOP-lowering medication. $^{76,77}$

ROCKs can be activated not only by Rho G-proteins, but also in response to lipids such as arachindonic acid. ${ }^{78}$ This may be the link between statin use and reduced glaucoma risk. ${ }^{77}$ However, the exact mechanism of how statins reduce the risk of glaucoma is not clear. Recently, an intronic variant in ARHGEF12 (Rho guanine nucleotide exchange factor (GEF) 12) was found to be associated with IOP and glaucoma, particularly in HTG. ${ }^{21}$ ARHGEF12 activates RhoA. ${ }^{79,80}$ Therefore, a possible mechanism might be that activation of RhoA via ARHGEF12 leads to ROCK activation, causing a decrease in AH outflow and IOP elevation (see Figure 5). This provides a link between common IOP variants and known IOP regulation mechanisms. Furthermore, it has been shown that ARHGEF12 can extend the half-life of the ABCA1 protein, ${ }^{81}$ known to be essential in the synthesis of HDL cholesterol. When ARHGEF12 binds to the $\mathrm{C}$ terminus of ABCA1 it activates RhoA that in turn prevents ABCA1 degradation. ${ }^{81}$ Moreover, analysis of proteins binding to APP, the first gene identified in Alzheimer's disease, demonstrated that both RhoA and GAS7 are part of the APP interactome. ${ }^{82}$ In addition, it has been shown that TGF- $\beta$ has an effect on GAS7 expression. ${ }^{83}$ As is the case for the CAV1/CAV2, ABCA1 and $A R G H E F 12$, variants in GAS7, have been found associated with both IOP and POAG, asking for further research evaluating the interaction between these proteins in the eye to elucidate the underlying mechanisms.

\section{Eye development}

There is strong evidence for the role of eye development genes in POAG, this is remarkable for a late-onset disorder. Eye development is a multistep process, starting with the formation of the optic vesicle that ultimately gives rise to the fully mature retina. Among essential transcription factors required for eye formation, $P A X 6$, ATOH7, and SIX6 have been associated with optic disc parameters, ${ }^{26,28,30,84}$ of which ATOH7 and SIX6 have also been associated with POAG.

Variants in ELP4 close to PAX6 were associated with DA in the Rotterdam Study. ${ }^{84} P A X 6$, a transcription factor considered as a master regulator gene in eye development, is required for lens differentiation and controls the expression of key regulatory transcription factors, such as ATOH7 and SIX6. ${ }^{85}$ In turn, ATOH7 is essential in the differentiation of the RGCs, the firstborn neurons in the retina. Studies have shown that ATOH7 plays a more important role in the determination of the DA than in the loss of RGC, ${ }^{29,31}$ the main characteristic of POAG. The relation between optic disc area and POAG is controversial. However, it has been suggested that individuals with larger optic discs may suffer more from IOP-related stress. ${ }^{86}$

SIX6 has been associated with VCDR and NTG. ${ }^{17,87}$ In zebrafish, knockdown of both zebrafish orthologs (six $6 a$ and six $6 b$ ) lead to small eye phenotype ${ }^{88,89}$ and possible abnormal differentiation of retinal progenitor cells. Moreover, it is known that SIX6 acts as transcriptional repressor of CDK inhibitors. Knockdown of six $6 b$ increases the expression of $c d k n 2 b$ in vivo. This not only provides a link between both loci but also between the eye development and TGF- $\beta$ pathway. ${ }^{88}$ In addition, variants in the antisense of SIX6 (SIX6-AS) have been associated with myopia, ${ }^{90}$ being together with $B M P 2$, one of the two overlapping loci between myopia and glaucoma. Myopia is a known risk factor for POAG, and further studies evaluating the genetic overlap between both conditions will extend the understanding of the genetic mechanism that determines the relation between myopia and glaucoma.

\section{Genes associated with the endophenotypes and their role in the reviewed pathways}

Table 1 shows the role of the loci associated with POAG endophenotypes in the context of the six biological processes discussed in this review (see pathways section). Of note is that genes may be attributed to multiple pathways, for example, $A B C A 1, C A V 1 / C A V 2$ (ECM and vascular tone), $C D K N 2 B-A S 1$, and $B M P 4$ (TGF- $\beta$ signalling and eye development). Genes associated with CCT seem to play an important role in ECM metabolism, whereas genes associated with optic disc parameters play a key role in eye development pathways. Genes involved in IOP are the major players in RhoA/ROCK signalling and vascular tone. It is noteworthy that many of the genes associated with POAG endophenotypes have been implicated in different type of cancers, in particular in gliomas. The relation between cancer and glaucoma seems stronger with genes associated with optic disc parameters, which are indicators of optic nerve degeneration; this is in line with emerging theories supporting the relation between neurodegeneration and cancer. ${ }^{91}$ Although neurodegeneration is caused by death of neurons and cancer cells are characterized by an 'immortal' phenotype, both are age-related conditions, and it has been observed that the same molecules play a role in processes such as cell proliferation, differentiation, 


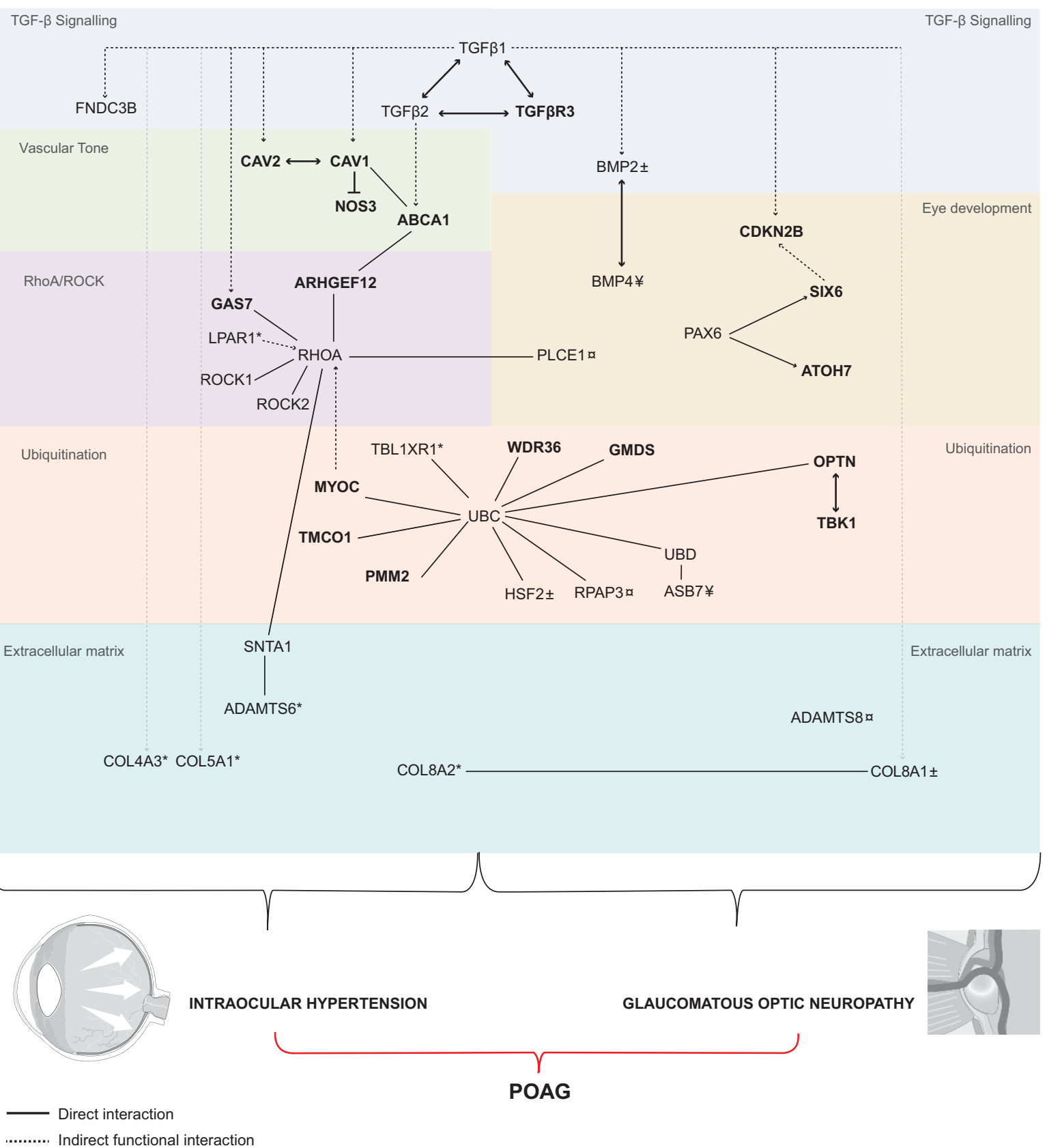

Figure 5 Pathways possibly implicated in POAG revealed by linkage and association studies. The six different biological processes possibly implicated in POAG reviewed are shown in the figure. Map was built using Ingenuity Pathway Analysis (IPA). Solid lines imply direct relationships between proteins (eg, physical protein-protein interaction or enzyme-substrate); dotted lines imply indirect functional relationships, such as coexpression, phosphorylation/dephosphorylation, activation/deactivation, transcription, or inhibition. Proteins in bold correspond to known glaucoma genes. Genes genome-wide associated with CCT $\left(^{*}\right)$, VCDR and CA $( \pm)$, CA only (¥), and VCDR only (a).

and apoptosis. It has been proposed that protein alterations that predispose cells to undergo apoptosis might lead to a decreased risk of cancer and increased risk of neurodegeneration, whereas protein modifications that facilitate cell growth might be protective for neurodegeneration but a risk factor for cancer. ${ }^{91}$
So far, at least six of the genes associated with VCDR, CA, or DA have been implicated in gliomas. These include the chromosome 9p21.3 locus in which CDKN2A, CDKN2B, and $C D K N 2 B-A S 1$ are located. This locus has also been associated with coronary artery disease, type 2 diabetes mellitus, and lymphoblastic leukaemia. ${ }^{92}$ 
Some variants are associated with various diseases beyond POAG. Variants in ABCA1, CAV1, and CAV2 have been associated with IOP and POAG; these genes are well known for their association with HDL cholesterol, atherogenenesis, and pulmonary hypertension, whereas variants in $A B O$ have been associated with IOP and also with venous thromboembolism, atherosclerosis, stroke, myocardial infraction, and diabetes. Furthermore, PAX6, known for its role in eye development, is also a key player in the development of the endocrine pancreas. Pax6 knockout mutants showed not only anophtalmia but also decreased production of insulin. ${ }^{93}$

\section{Building bridges between the various pathways identified in genetics research}

The six pathways discussed above may be linked to each other in several ways. Three out of the six pathways (TGF- $\beta$, RhoA/ROCK, and the Caveolins-1 and -2) have emerged as potential mechanism leading to the ECM changes observed in POAG or its early stages (endophenotypes). The TGF- $\beta$ signalling alters ECM production and turnover in both the TM and lamina cribrosa. Studies have demonstrated that high levels of TGF- $\beta 2$ promote a fibrotic phenotype in the TM. ${ }^{44,48}$ Interestingly, TGF $\beta$ R3 (associated with VCDR, DA, and POAG) is considered necessary for TGF- $\beta 2$ signal transduction. In addition, the RhoA/ROCK signalling has been also identified as significant player in the fibrogenic activity induced by TGF- $\beta 2 .{ }^{43,44,48}$ However, not only increased production of ECM proteins may lead to decreased outflow of $\mathrm{AH}$, but also alterations in their recycling and degradation may have this effect. CAV1 and CAV2 have been implicated in the endocytosis and degradation of ECM fibronectin, a glycoprotein of the ECM. ${ }^{40,41}$ Thus, dysregulation of CAV function could also contribute to the changes observed in the ECM in glaucoma. Furthermore, $C D K N 2 B$, one of the first genes implicated in POAG by GWAS and involved in cell cycle and growth regulation, is induced by TGF- $\beta .{ }^{53}$

Upregulation of $C D K N 2 B$ leads to a cell cycle arrest and has been found overexpressed in a rat model of glaucoma ${ }^{17}$ and in a six $6 b$ knockdown zebrafish model. ${ }^{88}$ TGF- $\beta$ has also been implicated in the activation of RhoA, as part of the non-smad signalling pathway. ${ }^{94}$ Both experimental and genetic data point to the TGF- $\beta$ signalling pathway as an important contributor to the complex pathogenesis of glaucoma. Figure 5 shows the different connections between the pathways emerging from combining the genetics and experimental findings.

Interestingly, two of the genes associated with POAG, GMDS (GDP-mannose 4,6-dehydratase) and PMM2 (phosphomannomutase 2), that are processed by the 
ubiquitin-proteasome system are also involved in the fructose and mannose metabolism. Mutations in PMM2 lead to congenital disorders involving a variety of clinical features related to the role of N-glycoproteins during embryonic development. These include defects in the nervous system development, cerebellar hypoplasia, peripheral neuropathy, as well as abnormal eye movement and retinitis pigmentosa. In POAG, the fructose and mannose pathways have also been found associated with disease status in a case/control study. ${ }^{95}$ A combination of genetics and metabolomics studies will help to clarify the role of carbohydrate metabolism in the pathophysiology of POAG.

There will be an important role of system biology and functional genomics research to disentangle the pathways. Systems biology aims to combine genomic, transcriptomic, proteomic, and metabolomic approaches along with computational models, whereas functional genomics aims to integrate multiple lines of evidence, for example, genetic data from human studies, gene expression, and animal models to identify, characterize, and prioritize candidate genes implicated in complex diseases. With the advent of GWASs, the amount of loci associated with glaucoma and its endophenotypes have increased significantly. So far, at least 70 loci have been associated with optic disc parameters, IOP, CCT, and POAG. Although these loci explain a small part of the variance in POAG endophenotypes, these loci have revealed and supported the role of several possible pathways implicated in the pathogenesis of POAG (discussed in this review). However, there is a lack of functional validation and detailed analysis of the in vivo function of these genes in the anterior chamber. More experimental data based on the animal models that are already available and new models are necessary.

There is a wide variety of animal models of different species in POAG, including monkeys, dogs, rabbits, rats, mice, chickens, and zebrafish. ${ }^{96}$ Different approaches have been used to study the possible molecular events leading to POAG. The first animal models in glaucoma focussed on non-human primates in which high IOP was induced, posteriorly high IOP was induced in other species, such as rabbits and mice. In addition, two other broad approaches have been used: (1) natural-occurring glaucoma models, or animal models with an induced glaucoma-like phenotype generated by the use of radiation or mutagens, in which subsequently the gene causing the phenotype is identified and (2) animal models in which a known candidate gene is mutated to evaluate the causal role of the gene or a specific mutation.

Among examples of the first category are: (1) an inherited form of POAG in dogs that led to the identification of mutations in ADAMTS10, which also belongs to the ADAMTS family, involved in ECM remodelling ${ }^{40}$ and
(2) analysis of the bugeye zebrafish led to the identification of a nonsense mutation in $\operatorname{lrp} 2$ as cause of a glaucoma-like phenotype, including severe myopia, high IOP, and progressive RGC death. Examples of the second category are: genetically modified animal models in which OPTN, MYOC, and WDR36 were either knockedout or mutated. ${ }^{97}$ These studies have helped in the understanding of the molecular events implicated in the development of glaucoma. Although the numbers of loci and candidate genes have increased dramatically in the past years, functional validation of the loci found associated with glaucoma needs to be performed to elucidate their role in the pathophysiology of the disease.

Zebrafish, for example, provides an excellent tool for high-throughput functional validation of GWASs loci. Recently, it has been demonstrated that substantial knockdown of six $6 a$ and six $6 b$ during early embryonic development causes a small eye phenotype. ${ }^{88,89}$ Analysis of the effect of six 6 depletion at later age as well will probably give more insight into the role of SIX6 in POAG and myopia.

Despite the fact that there are available mouse models for POAG genes such as cav1, abca1, and atoh7, and CCT genes such as col5a1 and colsa2, its role in the development of the disease has not been studied in depth yet. Although these have been developed for other diseases, they offer an opportunity to understand the pathogenesis of POAG-related phenotypes. For example, no studies focussed on IOP assessment in cav1 or abca1 mutants.

\section{Discussion}

In this paper we integrate findings from different organisms and different levels from molecular to (patho) physiological. We show that the genes involved in POAG and its endophenotypes can be linked to six pathways. These pathways can be studied jointly in cellular and animal models. There are still major gaps in our knowledge: many genes cannot be linked to the pathways that we proposed, and experimental studies will play a key role to link these genetic variants to the pathways described here or others. Whereas experimental studies show a key role of TGF- $\beta 2$, the genetic studies point to TGF $\beta$ R3. The genetic studies show evidence for a partly genetic overlapping aetiology with myopia (related to the eye development pathway), ALS, and Parkinson's and Alzheimer' disease (through ubiquitination pathway). This warrants off-target effect of therapeutic research addressing these disorders. Many genes that have been implicated in the ECM are not yet linked to POAG: more genetic studies are needed to do so. An important question is whether larger and more extensive studies will bring into view new pathways or whether these will fill in 
the missing genes in the pathways described here. We have not reached the state that we can answer this question. Without doubt, finding new variants will improve the translation of the genetic findings into clinical prediction and diagnosis.

Although to date the translation of genetic findings to clinical practice is limited, genetic findings will be of clinical value in the near future, particularly in the context of precision medicine, an emerging approach for prevention and disease treatment that takes into account individual variability in genes, environment, and lifestyle. ${ }^{98}$ In the case of POAG, strategies in precision medicine will aim to target, for instance, the specific pathways involved in the pathogenesis of POAG in each patient individually. A more in-depth knowledge of the pathways disturbed in glaucoma will help elucidating whether, for example, HTG patients have one particularly altered pathway, such as the RhoA/ROCK pathway, compared with NTG patients. This may change the treatment selection for these patients, who probably will benefit more from a selective ROCK inhibitor. Genetic profiling will be a time- and cost-effective approach to elucidate the underlying pathology. Further applications include the assessment of genetic variants related to drug response. Translation of these findings to clinical practice requires not only functional validation but also assessment of the clinical utility, that is, the use of test results (genetic test result) to inform clinical decision making. Knowledge integration across a wide range of biomedical fields is needed to translate genomic findings into clinical practice.

\section{Conflict of interest}

The authors declare no conflict of interest.

\section{References}

1 Tham YC, Li X, Wong TY, Quigley HA, Aung T, Cheng CY. Global prevalence of glaucoma and projections of glaucoma burden through 2040: a systematic review and metaanalysis. Ophthalmology 2014; 121(11): 2081-2090.

2 Fan BJ, Leung YF, Wang N, Lam SC, Liu Y, Tam OS et al. Genetic and environmental risk factors for primary openangle glaucoma. Chin Med J (Engl) 2004; 117(5): 706-710.

3 Zhao D, Cho J, Kim MH, Friedman DS, Guallar E. Diabetes, fasting glucose, and the risk of glaucoma: a meta-analysis. Ophthalmology 2015; 122(1): 72-78.

4 Ma F, Dai J, Sun X. Progress in understanding the association between high myopia and primary open-angle glaucoma. Clin Experiment Ophthalmol 2014; 42(2): 190-197.

5 Wolfs RC, Klaver CC, Ramrattan RS, van Duijn CM, Hofman A, de Jong PT. Genetic risk of primary open-angle glaucoma. Population-based familial aggregation study. Arch Ophthalmol 1998; 116(12): 1640-1645.

6 Fingert JH. Primary open-angle glaucoma genes. Eye (Lond) 2011; 25(5): 587-595.
7 Sanfilippo PG, Hewitt AW, Hammond CJ, Mackey DA. The heritability of ocular traits. Surv Ophthalmol 2010; 55(6): 561-583.

8 Stone EM, Fingert JH, Alward WL, Nguyen TD, Polansky JR, Sunden SL et al. Identification of a gene that causes primary open angle glaucoma. Science 1997; 275(5300): 668-670.

9 Rezaie T, Child A, Hitchings R, Brice G, Miller L, Coca-Prados $\mathrm{M}$ et al. Adult-onset primary open-angle glaucoma caused by mutations in optineurin. Science 2002; 295(5557): 1077-1079.

10 Monemi S, Spaeth G, DaSilva A, Popinchalk S, Ilitchev E, Liebmann $\mathrm{J}$ et al. Identification of a novel adult-onset primary open-angle glaucoma (POAG) gene on 5q22.1. Hum Mol Genet 2005; 14(6): 725-733.

11 Fingert JH, Robin AL, Stone JL, Roos BR, Davis LK, Scheetz TE et al. Copy number variations on chromosome $12 \mathrm{q} 14$ in patients with normal tension glaucoma. Hum Mol Genet 2011; 20(12): 2482-2494.

12 Ritch R, Darbro B, Menon G, Khanna CL, Solivan-Timpe F, Roos BR et al. TBK1 gene duplication and normal-tension glaucoma. JAMA Ophthalmol 2014; 132(5): 544-548.

13 Kawase K, Allingham RR, Meguro A, Mizuki N, Roos B, Solivan-Timpe FM et al. Confirmation of TBK1 duplication in normal tension glaucoma. Exp Eye Res 2012; 96(1): 178-180.

14 Awadalla MS, Fingert JH, Roos BE, Chen S, Holmes R, Graham SL et al. Copy number variations of TBK1 in Australian patients with primary open-angle glaucoma. Am J Ophthalmol 2015; 159(1): 124-30 e1.

15 Fan BJ, Wang DY, Lam DS, Pang CP. Gene mapping for primary open angle glaucoma. Clin Biochem 2006; 39(3): 249-258.

16 Thorleifsson G, Walters GB, Hewitt AW, Masson G, Helgason A, Dewan A et al. Common variants near CAV1 and CAV2 are associated with primary open-angle glaucoma. Nat Genet 2010; 42(10): 906-909.

17 Burdon KP, MacGregor S, Hewitt AW, Sharma S, Chidlow G, Mills RA et al. Genome-wide association study identifies susceptibility loci for open angle glaucoma at TMCO1 and CDKN2B-AS1. Nat Genet 2011; 43(6): 574-578.

18 Wiggs JL, Yaspan BL, Hauser MA, Kang JH, Allingham RR, Olson LM et al. Common variants at 9p21 and 8q22 are associated with increased susceptibility to optic nerve degeneration in glaucoma. PLoS Genet 2012; 8(4): e1002654.

19 Chen Y, Lin Y, Vithana EN, Jia L, Zuo X, Wong TY et al. Common variants near ABCA1 and in PMM2 are associated with primary open-angle glaucoma. Nat Genet 2014; 46(10): 1115-1119.

20 Gharahkhani P, Burdon KP, Fogarty R, Sharma S, Hewitt AW, Martin $\mathrm{S}$ et al. Common variants near ABCA1, AFAP1 and GMDS confer risk of primary open-angle glaucoma. Nat Genet 2014; 46(10): 1120-1125.

21 Springelkamp H, Iglesias AI, Cuellar-Partida G, Amin N, Burdon KP, van Leeuwen EM et al. ARHGEF12 influences the risk of glaucoma by increasing intraocular pressure. Hum Mol Genet 2015; 24(9): 2689-2699.

22 Wiggs JL, Kang JH, Yaspan BL, Mirel DB, Laurie C, Crenshaw A et al. Common variants near CAV1 and CAV2 are associated with primary open-angle glaucoma in Caucasians from the USA. Hum Mol Genet 2011; 20(23): 4707-4713.

23 Wiggs JL, Lynch S, Ynagi G, Maselli M, Auguste J, Del Bono EA et al. A genomewide scan identifies novel early- 
onset primary open-angle glaucoma loci on 9q22 and 20p12. Am J Hum Genet 2004; 74(6): 1314-1320.

24 Dimasi DP, Burdon KP, Hewitt AW, Fitzgerald J, Wang JJ, Healey PR et al. Genetic investigation into the endophenotypic status of central corneal thickness and optic disc parameters in relation to open-angle glaucoma. Am J Ophthalmol 2012; 154(5): 833-42 e2.

25 Li Z, Allingham RR, Nakano M, Jia L, Chen Y, Ikeda Y et al. A common variant near TGFBR3 is associated with primary open angle glaucoma. Hum Mol Genet 2015; 24(13): 3880-3892.

26 Ramdas WD, van Koolwijk LME, Ikram MK, Jansonius NM, de Jong PTVM, Bergen AAB et al. A genome-wide association study of optic disc parameters. PLoS Genet 2010; 6(6): e1000978.

27 Axenovich T, Zorkoltseva I, Belonogova N, van Koolwijk LM, Borodin $\mathrm{P}$, Kirichenko A et al. Linkage and association analyses of glaucoma related traits in a large pedigree from a Dutch genetically isolated population. J Med Genet 2011; 48 (12): 802-809.

28 Springelkamp H, Hohn R, Mishra A, Hysi PG, Khor CC, Loomis SJ et al. Meta-analysis of genome-wide association studies identifies novel loci that influence cupping and the glaucomatous process. Nat Commun 2014; 5: 4883.

29 Macgregor S, Hewitt AW, Hysi PG, Ruddle JB, Medland SE, Henders AK et al. Genome-wide association identifies ATOH7 as a major gene determining human optic disc size. Hum Mol Genet 2010; 19(13): 2716-2724.

30 Springelkamp H, Mishra A, Hysi PG, Gharahkhani P, Hohn R, Khor CC et al. Meta-analysis of genome-wide association studies identifies novel loci associated with optic disc morphology. Genet Epidemiol 2015; 39(3): 207-216.

31 Venturini C, Nag A, Hysi PG, Wang JJ, Wong TY, Healey PR et al. Clarifying the role of ATOH7 in glaucoma endophenotypes. Br J Ophthalmol 2014; 98(4): 562-566.

32 de Celis JF, Barrio R. Regulation and function of Spalt proteins during animal development. Int J Dev Biol 2009; 53 (8-10): 1385-1398.

33 Sunryd JC, Cheon B, Graham JB, Giorda KM, Fissore RA, Hebert DN. TMTC1 and TMTC2 are novel endoplasmic reticulum tetratricopeptide repeat-containing adapter proteins involved in calcium homeostasis. J Biol Chem 2014; 289(23): 16085-16099.

34 van Koolwijk LME, Ramdas WD, Ikram MK, Jansonius NM, Pasutto F, Hysi PG et al. Common genetic determinants of intraocular pressure and primary open-angle Glaucoma. PLoS Genet 2012; 8(5): e1002611.

35 Hysi PG, Cheng CY, Springelkamp H, Macgregor S, Bailey JNC, Wojciechowski R et al. Genome-wide analysis of multi-ancestry cohorts identifies new loci influencing intraocular pressure and susceptibility to glaucoma. Nat Genet 2014; 46(10): 1126-1130.

36 Nag A, Venturini C, Small KS, International Glaucoma Genetics Consortium, Young TL, Viswanathan AC et al. A genome-wide association study of intra-ocular pressure suggests a novel association in the gene FAM125B in the TwinsUK cohort. Hum Mol Genet 2014; 23(12): 3343-3348.

37 Lu Y, Vitart V, Burdon KP, Khor CC, Bykhovskaya Y, Mirshahi A et al. Genome-wide association analyses identify multiple loci associated with central corneal thickness and keratoconus. Nat Genet 2013; 45(2): 155-163.

38 Cai C, Rajaram M, Zhou X, Liu Q, Marchica J, Li J et al. Activation of multiple cancer pathways and tumor maintenance function of the $3 q$ amplified oncogene FNDC3B. Cell Cycle 2012; 11(9): 1773-1781.

39 Wang R, Wiggs JL. Common and rare genetic risk factors for glaucoma. Cold Spring Harb Perspect Med 2014; 4(12): a017244.

40 Vranka JA, Kelley MJ, Acott TS, Keller KE. Extracellular matrix in the trabecular meshwork: Intraocular pressure regulation and dysregulation in glaucoma. Exp Eye Res 2015; 133: $112-125$.

41 Wallace DM, Pokrovskaya O, O'Brien CJ. The function of matricellular proteins in the lamina cribrosa and trabecular meshwork in glaucoma. J Ocul Pharmacol Ther 2015; e-pub ahead of print 7 April 2015; doi:10.1089/jop.2014.0163.

42 Morales J, Al-Sharif L, Khalil DS, Shinwari JM, Bavi P, Al-Mahrouqi RA et al. Homozygous mutations in ADAMTS10 and ADAMTS17 cause lenticular myopia, ectopia lentis, glaucoma, spherophakia, and short stature. Am J Hum Genet 2009; 85(5): 558-568.

43 Fuchshofer R, Tamm ER. The role of TGF-(beta) in the pathogenesis of primary open-angle glaucoma. Cell Tissue Res 2012; 347(1): 279-290.

44 Wordinger RJ, Sharma T, Clark AF. The role of TGF-(beta)2 and bone morphogenetic proteins in the trabecular meshwork and glaucoma. J Ocul Pharmacol Ther 2014; 30(2-3): 154-162.

45 Jampel HD, Roche N, Stark WJ, Roberts AB. Transforming growth factor-beta in human aqueous humor. Curr Eye Res 1990; 9(10): 963-969.

46 Cousins SW, McCabe MM, Danielpour D, Streilein JW. Identification of transforming growth factor-beta as an immunosuppressive factor in aqueous humor. Invest Ophthalmol Vis Sci 1991; 32(8): 2201-2211.

47 Min SH, Lee TI, Chung YS, Kim HK. Transforming growth factor-beta levels in human aqueous humor of glaucomatous, diabetic and uveitic eyes. Korean J Ophthalmol 2006; 20(3): 162-165.

48 Fleenor DL, Shepard AR, Hellberg PE, Jacobson N, Pang IH, Clark AF. TGFbeta2-induced changes in human trabecular meshwork: implications for intraocular pressure. Invest Ophthalmol Vis Sci 2006; 47(1): 226-234.

49 Pena JD, Taylor AW, Ricard CS, Vidal I, Hernandez MR. Transforming growth factor beta isoforms in human optic nerve heads. Br J Ophthalmol 1999; 83(2): 209-218.

50 Quigley HA. Glaucoma. Lancet 2011; 377(9774): 1367-1377.

51 Wordinger RJ, Fleenor DL, Hellberg PE, Pang IH, Tovar TO, Zode GS et al. Effects of TGF-beta2, BMP-4, and gremlin in the trabecular meshwork: implications for glaucoma. Invest Ophthalmol Vis Sci 2007; 48(3): 1191-1200.

52 Barry RJ, Alsalem JA, Faassen J, Murray PI, Curnow SJ, Wallace GR. Association analysis of TGFBR3 gene with Behcet's disease and idiopathic intermediate uveitis in a Caucasian population. Br J Ophthalmol 2015; 99(5): 696-699.

53 Hannon GJ, Beach D. p15INK4B is a potential effector of TGF-beta-induced cell cycle arrest. Nature 1994; 371(6494): 257-261.

54 Tezel G. TNF-alpha signaling in glaucomatous neurodegeneration. Prog Brain Res 2008; 173: 409-421.

55 Cirulli ET, Lasseigne BN, Petrovski S, Sapp PC, Dion PA, Leblond CS et al. Exome sequencing in amyotrophic lateral sclerosis identifies risk genes and pathways. Science 2015; 347 (6229): 1436-1441.

56 Campello L, Esteve-Rudd J, Cuenca N, Martin-Nieto J. The ubiquitin-proteasome system in retinal health and disease. Mol Neurobiol 2013; 47(2): 790-810. 
57 Shen X, Ying H, Qiu Y, Park JS, Shyam R, Chi ZL et al. Processing of optineurin in neuronal cells. J Biol Chem 2011 286(5): 3618-3629.

58 Koga T, Shen X, Park JS, Qiu Y, Park BC, Shyam R et al. Differential effects of myocilin and optineurin, two glaucoma genes, on neurite outgrowth. Am J Pathol 2010; 176(1): 343-352.

59 Qiu Y, Shen X, Shyam R, Yue BY, Ying H. Cellular processing of myocilin. PLoS One 2014; 9(4): e92845.

60 Minegishi Y, Iejima D, Kobayashi H, Chi ZL, Kawase K, Yamamoto $T$ et al. Enhanced optineurin E50K-TBK1 interaction evokes protein insolubility and initiates familial primary open-angle glaucoma. Hum Mol Genet 2013; 22(17) 3559-3567.

61 Hewitt AW, Mackey DA, Craig JE. Myocilin allele-specific glaucoma phenotype database. Hum Mutat 2008; 29(2): 207-211.

62 Anholt RR, Carbone MA. A molecular mechanism for glaucoma: endoplasmic reticulum stress and the unfolded protein response. Trends Mol Med 2013; 19(10): 586-593.

63 Janssen SF, Gorgels TG, Ramdas WD, Klaver CC, van Duijn CM, Jansonius NM et al. The vast complexity of primary open angle glaucoma: disease genes, risks, molecular mechanisms and pathobiology. Prog Retin Eye Res 2013; 37: 31-67.

64 Venkataraman ST, Flanagan JG, Hudson C. Vascular reactivity of optic nerve head and retinal blood vessels in glaucoma-a review. Microcirculation 2010; 17(7): 568-581.

65 Nathanson JA, McKee M. Alterations of ocular nitric oxide synthase in human glaucoma. Invest Ophthalmol Vis Sci 1995 36(9): 1774-1784.

66 Stamer WD, Lei Y, Boussommier-Calleja A, Overby DR, Ethier CR. eNOS, a pressure-dependent regulator of intraocular pressure. Invest Ophthalmol Vis Sci 2011; 52(13): 9438-9444.

67 Kang JH, Wiggs JL, Rosner BA, Hankinson SE, Abdrabou W, Fan BJ et al. Endothelial nitric oxide synthase gene variants and primary open-angle glaucoma: interactions with sex and postmenopausal hormone use. Invest Ophthalmol Vis Sci 2010; 51(2): 971-979.

68 Logan JF, Chakravarthy U, Hughes AE, Patterson CC, Jackson JA, Rankin SJ. Evidence for association of endothelial nitric oxide synthase gene in subjects with glaucoma and a history of migraine. Invest Ophthalmol Vis Sci 2005; 46(9): 3221-3226.

69 Tunny TJ, Richardson KA, Clark CV. Association study of the 5 ' flanking regions of endothelial-nitric oxide synthase and endothelin-1 genes in familial primary open-angle glaucoma. Clin Exp Pharmacol Physiol 1998; 25(1): 26-29.

70 Li X, McClellan ME, Tanito M, Garteiser P, Towner R, Bissig D et al. Loss of caveolin-1 impairs retinal function due to disturbance of subretinal microenvironment. J Biol Chem 2012; 287(20): 16424-16434.

$71 \mathrm{Gu}$ X, Fliesler SJ, Zhao YY, Stallcup WB, Cohen AW, Elliott MH. Loss of caveolin-1 causes blood-retinal barrier breakdown, venous enlargement, and mural cell alteration. Am J Pathol 2014; 184(2): 541-555.

72 Rahman A, Sward K. The role of caveolin-1 in cardiovascular regulation. Acta Physiol (Oxf) 2009; 195(2): 231-245.

73 Kuo CY, Lin YC, Yang JJ, Yang VC. Interaction abolishment between mutant caveolin-1(Delta62-100) and ABCA1 reduces HDL-mediated cellular cholesterol efflux. Biochem Biophys Res Commun 2011; 414(2): 337-343.
74 Fukata Y, Amano M, Kaibuchi K. Rho-Rho-kinase pathway in smooth muscle contraction and cytoskeletal reorganization of non-muscle cells. Trends Pharmacol Sci 2001; 22(1): 32-39.

75 Honjo M, Tanihara H, Inatani M, Kido N, Sawamura T, Yue BY et al. Effects of rho-associated protein kinase inhibitor Y-27632 on intraocular pressure and outflow facility. Invest Ophthalmol Vis Sci 2001; 42(1): 137-144.

76 Inoue T, Tanihara H. Rho-associated kinase inhibitors: a novel glaucoma therapy. Prog Retin Eye Res 2013; 37: $1-12$.

77 Wang SK, Chang RT. An emerging treatment option for glaucoma: Rho kinase inhibitors. Clin Ophthalmol 2014; 8: 883-890.

78 Feng J, Ito M, Kureishi Y, Ichikawa K, Amano M, Isaka N et al. Rho-associated kinase of chicken gizzard smooth muscle. J Biol Chem 1999; 274(6): 3744-3752.

79 Aittaleb M, Gao G, Evelyn CR, Neubig RR, Tesmer JJ. A conserved hydrophobic surface of the LARG pleckstrin homology domain is critical for RhoA activation in cells. Cell Signal 2009; 21(11): 1569-1578.

80 Lessey-Morillon EC, Osborne LD, Monaghan-Benson E, Guilluy C, O'Brien ET, Superfine R et al. The RhoA guanine nucleotide exchange factor, LARG, mediates ICAM-1dependent mechanotransduction in endothelial cells to stimulate transendothelial migration. J Immunol 2014; 192(7): 3390-3398.

81 Okuhira K, Fitzgerald ML, Tamehiro N, Ohoka N, Suzuki K, Sawada J et al. Binding of PDZ-RhoGEF to ATP-binding cassette transporter A1 (ABCA1) induces cholesterol efflux through RhoA activation and prevention of transporter degradation. J Biol Chem 2010; 285(21): 16369-16377.

82 Bai Y, Markham K, Chen F, Weerasekera R, Watts J, Horne P et al. The in vivo brain interactome of the amyloid precursor protein. Mol Cell Proteomics 2008; 7(1): 15-34.

83 Chang Y, Ueng SW, Lin-Chao S, Chao CC. Involvement of Gas7 along the ERK1/2 MAP kinase and SOX9 pathway in chondrogenesis of human marrow-derived mesenchymal stem cells. Osteoarthritis Cartilage 2008; 16(11): 1403-1412.

84 Gasten AC, Ramdas WD, Broer L, van Koolwijk LM, Ikram MK, de Jong PT et al. A genetic epidemiologic study of candidate genes involved in the optic nerve head morphology. Invest Ophthalmol Vis Sci 2012; 53(3): 1485-1491.

85 Ashery-Padan R, Gruss P. Pax6 lights-up the way for eye development. Curr Opin Cell Biol 2001; 13(6): 706-714.

86 Bellezza AJ, Hart RT, Burgoyne CF. The optic nerve head as a biomechanical structure: initial finite element modeling. Invest Ophthalmol Vis Sci 2000; 41(10): 2991-3000.

87 Pasquale LR, Loomis SJ, Kang JH, Yaspan BL, Abdrabou W, Budenz DL et al. CDKN2B-AS1 genotype-glaucoma feature correlations in primary open-angle glaucoma patients from the United States. Am J Ophthalmol 2013; 155(2): 342-53 e5.

88 Iglesias AI, Springelkamp H, Van der linde H, Severijnen L, Amin N, Oostra B et al. Exome sequencing and functional analyses suggest that SIX6 is a gene involved in an altered proliferation-differentiation balance early in life and optic nerve degeneration at old age. Hum Mol Genet 2014; 23(5): 1320-1332.

89 Carnes MU, Liu YP, Allingham RR, Whigham BT, Havens S, Garrett ME et al. Discovery and functional annotation of SIX6 variants in primary open-angle glaucoma. PLoS Genet 2014; 10(5): e1004372. 
90 Verhoeven VJ, Hysi PG, Wojciechowski R, Fan Q, Guggenheim JA, Hohn R et al. Genome-wide meta-analyses of multiancestry cohorts identify multiple new susceptibility loci for refractive error and myopia. Nat Genet 2013; 45(3): 314-318.

91 Plun-Favreau H, Lewis PA, Hardy J, Martins LM, Wood NW. Cancer and neurodegeneration: between the devil and the deep blue sea. PLoS Genet 2010; 6(12): e1001257.

92 Holdt LM, Teupser D. Recent studies of the human chromosome 9p21 locus, which is associated with atherosclerosis in human populations. Arterioscler Thromb Vasc Biol 2012; 32(2): 196-206.

93 Nitta Y, Shigeyoshi Y, Nakagata N, Kaneko T, Nitta K, Harada $\mathrm{T}$ et al. Kinetics of blood glucose in mice carrying hemizygous Pax6. Exp Anim 2009; 58(2): 105-112.
$94 \mathrm{Mu}$ Y, Gudey SK, Landstrom M. Non-Smad signaling pathways. Cell Tissue Res 2012; 347(1): 11-20.

95 Roberson RM, Uppal K, Burgess LG, Tran V, Kuchtey J, Kuchtey RW et al. Metabolic profiles associated with primary open-angle glaucoma. Invest Ophthalmol Visual Sci 2014; 55(13): 5703.

96 Bouhenni RA, Dunmire J, Sewell A, Edward DP. Animal models of glaucoma. J Biomed Biotechnol 2012; 2012: 692609.

97 Veth KN, Willer JR, Collery RF, Gray MP, Willer GB, Wagner DS et al. Mutations in zebrafish lrp2 result in adult-onset ocular pathogenesis that models myopia and other risk factors for glaucoma. PLoS Genet 2011; 7(2): e1001310.

98 Jameson JL, Longo DL. Precision medicine-personalized, problematic, and promising. N Engl J Med 2015; 372(23): 2229-2234.

Supplementary Information accompanies this paper on Eye website (http://www.nature.com/eye) 